\title{
Tuberculosis del sistema nervioso central en una paciente anciana: reporte de caso

\author{
Alejandra Palacio Sanguino ${ }^{\mathrm{a}}$ \\ Unidad de Geriatría, Hospital Universitario \\ San Ignacio, Bogotá, Colombia. Instituto de \\ Envejecimiento, Facultad de Medicina de la Pontificia \\ Universidad Javeriana, Bogotá, Colombia \\ Carlos José Ariza Galindo \\ Unidad de Geriatría, Hospital Universitario \\ San Ignacio, Bogotá, Colombia. Instituto de \\ Envejecimiento, Facultad de Medicina de la Pontificia \\ Universidad Javeriana, Bogotá, Colombia \\ Ronald Camilo Gómez Arteaga \\ Unidad de Geriatría, Hospital Universitario \\ San Ignacio, Bogotá, Colombia. Instituto de \\ Envejecimiento, Facultad de Medicina de la Pontificia \\ Universidad Javeriana. Departamento Medicina \\ Interna y Geriatría, Hospital Cardiovascular Soacha, \\ Cundinamarca, Colombia. Departamento de Medicina \\ Interna y Geriatría, Clínica del Country, Bogotá,
}

Colombia

${ }^{\text {a }}$ Autora de correspondencia: alejandrapalacio@javeriana.edu.co

Cómo citar: Palacio Sanguino A, Ariza Galindo CJ, Gómez Arteaga RC. Tuberculosis del sistema nervioso central de una paciente anciana: reporte de caso. Univ. Med. 2020;61(4). https://doi.org/10.11144/Jave riana.umed61-4.tsnc

\section{RESUMEN}

La tuberculosis (TB) es una patología prevalente en nuestra sociedad, y la población anciana es una de las más susceptibles a desarrollarla, dada la presencia de condiciones especiales, como inmunosenescencia, pluripatología, polifarmacia, desnutrición, entre otros. Se presenta el caso de una mujer anciana que ingresó al servicio de urgencias por deterioro clínico secundario a alteración del estado de conciencia en quien se descartaron causas principales como traumatismo craneoencefálico, sangrado o eventos isquémicos cerebrales y estado convulsivo. Al reinterrogarla se encontraron síntomas respiratorios de larga data con hallazgos radiográficos de $\mathrm{TB}$, confirmados por tomografía de tórax, con hallazgos posteriores de neuroinfección, dada la persistencia de alteración del estado de conciencia y el reporte de la punción lumbar.

Palabras clave

tuberculosis; anciano; meningitis; tuberculoma.

\begin{abstract}
Tuberculosis (TB) is a pathology prevalent at a global level in our society, the elderly population being one of the most susceptible to develop it given the presence of special conditions such as: immunosenescence, pluripatology, polypharmacy, malnutrition, among others. The case of an elderly patient who is admitted to the emergency department due to clinical deterioration secondary to altered state of consciousness
\end{abstract}


is presented below. Primary causes such as TBI, bleeding or cerebral ischemic events and seizure status are ruled out. Upon interrogation, long-standing respiratory symptoms were found with radiographic findings of tuberculosis, confirmed by chest CT, with subsequent neuroinfection findings given the persistence of altered state of consciousness and the report of lumbar puncture.

Keywords

tuberculosis; aged; meningitis; tuberculoma.

\section{Introducción}

La tuberculosis (TB) sigue siendo un problema de salud pública en Colombia. Para 2018 se notificaron al sistema de vigilancia (Sivigila) 14.446 casos de todas las formas, 13.032 nuevos y 389 recaídas. La tasa de notificación de TB en todas sus formas fue de 26,9 casos por cada 100.000 habitantes. La infección pulmonar corresponde a 22,1 casos por cada 100.000 habitantes y la extrapulmonar a 4,8 casos por 100.000 habitantes (1). En los últimos 6 años, los casos notificados presentan un aumento de 23 casos por 100.000 habitantes en 2013 hasta 26,9 casos por cada 100.000 habitantes en 2018 (1).

Una de las presentaciones de TB más grave es el compromiso del sistema nervioso central, dado principalmente por meningitis, cuya mortalidad es superior al $50 \%$, y por la alta probabilidad de secuelas neurológicas graves, a pesar del tratamiento médico, en gran parte debido al diagnóstico tardío $(2,3)$.

En la población geriátrica hay un mayor riesgo de infección por Mycobacteriumtuberculosis, debido a factores biológicos como malnutrición, estado inmunitario deficiente, comorbilidades superpuestas, uso crónico de medicamentos y factores sociodemográficos (por ejemplo, pobreza, condiciones de vida o acceso a la atención médica) (4). A continuación, presentamos un caso de TB meníngea en una mujer anciana hospitalizada en una unidad de cuidados agudos de geriatría.

\section{Descripción del caso}

El caso corresponde a una mujer de 80 años de edad, quien era independiente para actividades básicas e instrumentales de la vida diaria, sin trastorno neurocognoscitivo mayor, sin antecedentes patológicos, ni farmacológicos de importancia. Ingresó traída por familiares por un cuadro clínico de un día de evolución, caracterizado por alteración del estado de conciencia, asociado a desorientación en tiempo, espacio y persona; lenguaje incoherente; disminución de fuerza en las extremidades; relajación de esfínteres, y posterior caída desde su altura, sin ser clara la presencia de trauma craneoencefálico o movimientos anormales.

Fue llevada al servicio de urgencias, donde decidieron vigilarla en la unidad de reanimación, ante el deterioro del estado de conciencia $\mathrm{y}$, posteriormente, trasladarla a la unidad de cuidados intensivos. Se le realizó una tomografía de cráneo, que descartó lesiones traumáticas agudas, con hallazgos de microangiopatía isquémica crónica, sin sangrado o alteraciones isquémicas. $\mathrm{Al}$ efectuarle la bioquímica hemática, se encontró hiponatremia severa (120 mmol/l) e hipocloremia ( $88 \mathrm{mmol} / \mathrm{l})$, con sospecha diagnóstica de síndrome de secreción inadecuada de la hormona antidiurética (SIADH) con exámenes paraclínicos que evidenciaban osmolaridad sérica de $248 \mathrm{mOsm} /$ $\mathrm{kg}$; sodio en orina de $171 \mathrm{mEq} / \mathrm{L}$ (mayor de $40 \mathrm{mEq} / \mathrm{L}$ ), y osmolaridad urinaria de 577,814 $\mathrm{mOsm} / \mathrm{kg}$ con persistencia de hiponatremia severa y alteración del estado de conciencia a pesar de las intervenciones. Se reinterrogó a los familiares y se halló que, previo al ingreso, la paciente cursaba con síntomas respiratorios inespecíficos. Se le solicitó una radiografía de tórax con evidencia de infiltración intersticial de patrón micronodular en el parénquima pulmonar bilateral sugestiva de patrón miliar por TB (figura 1A). 
Figura 1.

A) Radiografía de tórax. B) Tomografía contrastada de tórax. C) Resonancia magnética de cerebro, simple y contrastada

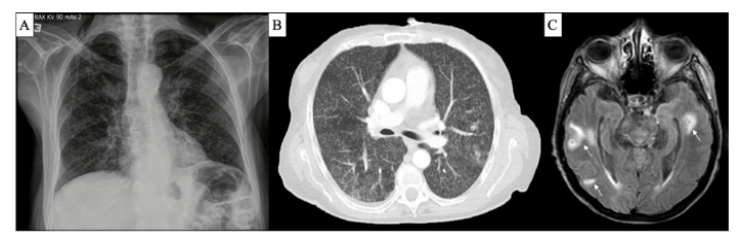

Se decidió complementar los estudios con una tomografía de tórax contrastada y baciloscopias, ante la sospecha de infección por TB. Esta imagen evidenció innumerables nódulos pulmonares de distribución aleatoria en ambos pulmones, con medidas que oscilan entre 1 y $3 \mathrm{~mm}$. Existían otros nódulos pulmonares de mayor tamaño y localización, principalmente subpleural con densidad de tejidos blandos y consolidación en el segmento apicoposterior del lóbulo superior izquierdo, con granuloma calcificado en el lóbulo inferior derecho, y con ello se consideraron los hallazgos descritos en el parénquima pulmonar, sugestivos de TB miliar (figura 1B).

Se le solicitó una valoración al servicio de neumología, que consideró oportuno iniciar un esquema antituberculosis con tetraconjugado (rifampicina de $150 \mathrm{mg}+$ isonazida de $75 \mathrm{mg}$ + pirazinamida de $400 \mathrm{mg}+$ etambutol de $275 \mathrm{mg}$ por día). También se le realizó una fibrobroncoscopia con biopsia transbronquial (con reporte de baciloscopias negativas, estudio citopatológico sin evidencia de granulomas ni de hallazgos sugestivos de malignidad y cultivo de lavado broncoalveolar negativo).

La mujer fue trasladada a sala general por presentar una leve mejoría; sin embargo, persistía con alteración del estado de conciencia y alteración hidroelectrolítica, a pesar del manejo establecido. Ello hizo que la valorara el servicio de neurología, que le efectuó punción lumbar con resultado de estudio citoquímico de líquido cefalorraquídeo con pleocitosis neutrofílica, hipoglucorraquia e hiperproteinemia (leucocitos: 95 células; neutrófilos: $80 \%$; hematíes: 2 células; frescos: $100 \%$; glucosa: $27 \mathrm{mg} / \mathrm{dl}$; proteínas: 173,23 $\mathrm{mg} / \mathrm{dL})$, tinciones microbiológicas negativas, reacción en cadena de la polimerasa positiva para TB (GenXpert Ultra) y prueba ADA positiva (6,72 U/L), hallazgos compatibles con meningitis tuberculosa en un paciente VIH negativo.

Se le adicionó manejo con corticoide sistémico (dexametasona endovenosa con descenso progresivo semanal durante 4 semanas). También se le tomó una resonancia magnética de cerebro, simple y contrastada, que evidenció mútiples lesiones focales de centro hipointenso en T2, con realce periférico después de la inyección del gadolinio, dispersas en las cortezas de ambos hemisferios cerebrales y del cerebelo, así como en el brazo posterior de la cápsula interna derecha, en el aspecto lateral del hipotálamo derecho y en el aspecto anteromedial izquierdo del mesencéfalo, con diámetros que oscilaron entre 2 y $8 \mathrm{~mm}$, con escaso a moderado edema circundante. Se visualizaron zonas de realce leptomenígeo en la porción basal medial del lóbulo frontal izquierdo y en la superficie lateral derecha del bulbo raquídeo, considerando que los hallazgos son atribuibles como primera posibilidad a infección meníngea y encefálica por TB con múltiples tuberculomas (figura 1C).

Posterior al manejo establecido, se observó una mejoría importante en la clínica y la funcionalidad de la paciente. Se evidenció una incorporación en sus actividades básicas de la vida diaria, se inició terapia física intrahospitalaria y se ajustó su dieta, por mostrar desnutrición. La paciente egresó deambulando por sus propios medios con recuperación parcial de su funcionalidad (Barthel de egreso: 65/100 vs. Barthel de ingreso: 0/100).

\section{Discusión}

El compromiso del sistema nervioso central por TB incluye la meningitis tuberculosa y los tuberculomas cerebrales, los cuales corresponden a un reto diagnóstico para el clínico (2). Generalmente, la meningitis tuberculosa se presenta como una meningitis subaguda o crónica, lo que ocurre aproximadamente entre 7 y 30 días caracterizados por cefalea, picos 
febriles y emesis, que culmina en pérdida del estado de conciencia, déficit neurológico focal o muerte (5). Su diagnóstico es clínico, con confirmación por microscopia y cultivo de líquido cefalorraquídeo o reacción en cadena de la polimerasa (6). En la literatura sobre el tema se ha descrito que detecta significativamente más meningitis tuberculosa que Xpert o cultivo, motivo por el cual la Organización Mundial de la Salud recomienda su uso como prueba de diagnóstico inicial ante la sospecha de meningitis tuberculosa (7).

Epidemiológicamente, la TB meníngea representa el $1 \%$ de todos los casos de TB y aproximadamente el $5 \%$ de los casos de TB extrapulmonar (3). La meníngea afecta a todos los grupos de edad, pero es mucho más frecuente observarla en niños y pacientes coinfectados con $\mathrm{VIH}$, quienes tienen hasta 5 veces más riesgo de desarrollarla en el sistema nervioso central (8). En la población geriátrica aún se desconoce la incidencia de esta enfermedad para Colombia y el mundo.

Las causas de muerte y discapacidad en la meningitis tuberculosa son multifactoriales y los principales mecanismos patológicos son persistentes, como la presión intracraneal elevada progresiva con hidrocefalia o sin esta, compromiso de los nervios ópticos o quiasma óptico que conduce a déficit visual, neuropatías craneales, aracnoiditis y vasculitis de los vasos sanguíneos cerebrales que conducen a un derrame cerebral, sin perder de vista las posibles complicaciones asociadas al manejo antituberculoso y su toxicidad asociada (6).

La tuberculosis meníngea se clasifica en tres estadios: estadio I (leve), sin alteración de la conciencia o signos de focalización neurología focal; estadio II (casos moderadamente avanzados), alteración del estado de conciencia pero sin coma y aquellos con déficits neurológicos (por ejemplo, parálisis de un solo nervio craneal, paraparesia y hemiparesias), y estadio III (casos graves), deterioro severo del sistema nervioso, coma y aquellos con múltiples parálisis de nervios craneales, hemiplejia o paraplejia, o ambas (6). El caso clínico actual se encontraba en un estadio III. Otros hallazgos paraclínicos relevantes en nuestro caso fueron la hiponatremia asociada, que se describe hasta en el $44,7 \%$ de los pacientes con meningitis tuberculosa, cuyo comportamiento clínico corresponde a un grado de moderado a severo en al menos el $90 \%$ de los casos asociado a SIADH y a cerebro perdedor de sal (9). El tratamiento actual se ha enfocado en recibir esquemas antituberculosos con: isoniazida, rifampicina, pirazinamida y etambutol. Estos casos presentan una modesta mejoría de supervivencia comparado a no recibirlos; sin embargo, la mortalidad permanece alta (6).

Existen esquemas de tratamiento adyuvante con dexametasona con evidencia de mejoría de la supervivencia en mayores de 14 años con meningitis tuberculosa; pero probablemente sin disminución en una discapacidad grave (10). Pueden disminuir la inflamación, especialmente en el espacio subaracnoideo, reducir el edema cerebral de la médula espinal y la presión intracraneal (10), además de reducir la inflamación de pequeños vasos sanguíneos y daños debidos a la disminución del flujo sanguíneo (6). Como ya se describió, la paciente recibió manejo con terapia antituberculosa; sin embargo, los cambios significativos fueron mucho más evidentes después del inicio de la terapia con corticoides endovenosos, y de manera concomitante la optimización del estado nutricional y el inicio precoz e intensivo de estrategias de rehabilitación física.

\section{Responsabilidades éticas}

Protección de personas y animales. Los autores declaran que para este reporte de caso no se realizaron experimentos en seres humanos ni en animales.

Confidencialidad de los datos. Los autores declaran que en este artículo no aparecen datos que permitan identificar al paciente.

Derecho a la privacidad y consentimiento informado. Los autores han obtenido el consentimiento informado del paciente referido en el artículo. Este documento obra en poder del autor de correspondencia. 


\section{Financiación}

Vicerrectoría de Investigaciones de la Pontificia Universidad Javeriana (Bogotá), Dirección científica del Hospital Universitario San Ignacio.

\section{Conflicto de intereses}

Los autores declaran no tener ningún conflicto de intereses.

\section{Referencias}

1. Instituto Nacional de Salud. Informe de evento: tuberculosis Colombia 2019 [internet]; 2019 may 31. Disponible en: http://www.ins.gov.co/buscador-ev entos/Informesdeevento/TUBERCUL OSIS SEMESTRE I 2019.pdf

2. Méchaï F, Bouchaud O. Tuberculous meningitis: challenges in diagnosis and management. Rev Neurol (Paris). 2019;175(7-8):451-7. https://doi.org/1 0.1016/j.neurol.2019.07.007

3. Thwaites GE, van Toorn R, Schoeman J. Tuberculous meningitis: more questions, still too few answers. Lancet Neurol. 2013;12(10):999-1010.

4. Rajagopalan S. Tuberculosis in older adults. Clin Geriatr Med. 2016;32 (3):479-91. https://doi.org/10. 1016/j.cger.2016.02.006

5. Mai NT, Thwaites GE. Recent advances in the diagnosis and management of tuberculous meningitis. Curr Opin Infect Dis. 2017;30(1):123-8. https://doi.org/10.1 097/QCO.0000000000000331
6. Prasad K SM, Ryan $\mathrm{H}$. Corticosteroids for managing tuberculous meningitis [review]. Cochrane Database Syst Rev. 2016 Apr 28;4(4):CD002244. https://doi.org/10. 1002/14651858.CD002244.pub4.

7. Bahr NC, Nuwagira E, Evans EE, Cresswell FV, Bystrom PV, Byamukama A, et al. Diagnostic accuracy of Xpert MTB/RIF Ultra for tuberculous meningitis in HIV-infected adults: a prospective cohort study. Lancet Infect Dis. 2018;18(1):68-75. https://doi.org/ 10.1016/S1473-3099(17)30474-7

8. Cecchini D, Ambrosioni J, Brezzo C, Corti M, Rybko A, Perez $\mathrm{M}$, et al. Tuberculous meningitis in HIV-infected and non-infected patients: comparison of cerebrospinal fluid findings. Int J Tuberc Lung Dis [internet]. 2009;13(2):269-71. Disponible en: https://www.ingentacon nect.com/content/iuatld/ijtld/2009/00 000013/00000002/art00020;jsessionid $=18 \mathrm{j} 4 \mathrm{amfvj} 9 \mathrm{~g} 9 \mathrm{u} \cdot \mathrm{x}$-ic-live-03

9. Misra UK, Kalita J, Bhoi SK, Singh RK. A study of hyponatremia in tuberculous meningitis. J Neurol Sci. 2016;367:152-7. https://doi.org/10.101 6/j.jns.2016.06.004

10. Thwaites GE, Nguyen DB, Nguyen HD, Hoang TQ, Do TT, Nguyen TC, et al. Dexamethasone for the treatment of tuberculous meningitis in adolescents and adults. N Engl J Med. 2004;351(17):1741-51. https://doi.org/ 10.1056/NEJMoa040573 